\title{
Synthesis of new thiosulfonate derivatives with quinone and quinoxaline fragments
}

\author{
K. Bolibrukh, N. Monka, S. Polovkovych, V. Lubenets, V. Novikov \\ Department of Technology of Biologically Active Substances, Pharmacy and Biotechnology, \\ National University "Lviv Polytechnic", \\ 79013, Ukraine, Lviv, Bandera Str. 12 \\ E-mailvnovikov@polynet.lviv.ua
}

\section{O. Khoumeri, T. Terme, P. Vanelle}

Aix-Marseille Univ, CNRS, Institut de Chimie Radicalaire ICR, UMR 7273,

Laboratoire de Pharmaco-Chimie Radicalaire, Faculté de Pharmacie,

13385, France, Marseille Cedex 05, Bd J. Moulin, 27

E-maillpcr@pharmacie.univ.mrs.fr

\section{O. Solovyov}

Shupyk National Medical Academy of Postgraduate Education, 04112, Kyiv, Dorohozhyts'ka Str. 9, Ukraine

crossref http://dx.doi.org/10.5755/j01.ct.64.2.6019

Received 27 November 2013; Accepted 18 December 2013

\begin{abstract}
The optimisation of reaction conditions for obtaining thiosulfonate derivatives was performed. S,N-binucleophiles (sodium salts of 4-amino- and 4-acetylaminobenzenethiosulfonic acids) were used. The parameters of the reaction for the synthesis of derivatives by a thiosulfonate fragment were determined. Thiosulfonate derivatives based on a number of quinones and quinoxalines were synthesized.
\end{abstract}

Keywords: thiosulfonates, binucleophile, quinone, quinoxaline, nucleophilic substitution

\section{Introduction}

Thiosulfonates and their derivatives are known to possess a wide spectrum of antimicrobial activity and also are used as fungicides and bacteriocides [1-8]. Quinone and its derivatives - the natural and synthetic compounds - play a considerable role as they are involved in photosynthesis and are the moiety of some vitamins. The efficacy of anthracycline antibiotics, such as daunomycin and adriamycin, in the treatment of a variety of human malignancies has stimulated a continued interest in the synthesis of this antitumor class agents [9, 10]. Likewise, quinoxaline derivatives show very interesting biological properties $[11,12]$ such as antibacterial, antiviral, anticancer, antifungal, anti-HIV, anti-inflammatory.

The aforementioned properties and clinical significance of these classes of compounds have stimulated the synthesis of new lead compounds with the expected broad spectrum of properties.

\section{Materials and methods}

Melting points were determined on a Büchi capillary melting point apparatus and are uncorrected. Element analyses were performed by the centre of Microanalyse of the Aix-Marseille University. Both ${ }^{1} \mathrm{H}$ and ${ }^{13} \mathrm{C}$ NMR spectra were determined on a Bruker AC 200 spectrometer. The ${ }^{1} \mathrm{H}$ and ${ }^{13} \mathrm{C}$ chemical shifts are reported from $\mathrm{CDCl}_{3}$ peaks: ${ }^{1} \mathrm{H}(7.26 \mathrm{ppm})$ and ${ }^{13} \mathrm{C}(77.16 \mathrm{ppm})$, and from DMSO- $d_{6}$ peaks: ${ }^{1} \mathrm{H}(2.50 \mathrm{ppm})$ and ${ }^{13} \mathrm{C}$ (39.52 ppm).

The following adsorbents were used for column chromatography: silica gel 60 (Merck, particle size 0.063-0.200 mm, 70-230 mesh ASTM). TLC was performed on $5 \mathrm{~cm} \times 10 \mathrm{~cm}$ aluminium plates coated with silica gel 60 F254 (Merck) in an appropriate solvent.

General procedure for the synthesis of 4-aminobenzenethiosulfonic acid S-(R-methyl) esters 3 a-i

Into a two-necked flask equipped with a nitrogen inlet, a solution of the appropriate methylhalogenated derivatives $\mathbf{1} \mathbf{a}-\mathbf{i}$ in THF and dissolved in a portion of THF sodium salt of 4-aminobenzenethiosulfonic acid (2 a) were added. The solution was stirred and kept at room temperature for 5 hours. After this time, a TLC analysis showed that compound 2 had been totally consumed. The reaction mixture was treated with ice water and extracted 3 times with dichloromethane. The organic phase was washed with water and then dried over anhydrous sodium sulfate. After evaporation, the product purified by silica gel chromatography and recrystallized from ethanol gave the corresponding 4-aminobenzenethiosulfonic acid S-(R-methyl) esters 3 a-i. 
By these procedure compounds $\mathbf{3}$ a-i were synthesized.

4-Aminobenzenethiosulfonic acid S-(4-nitrobenzyl) ester (3 a)

Yellow precipitate, Mp. $273-276{ }^{\circ} \mathrm{C}$, yield $63 \%$.

${ }^{1} \mathrm{H}$ NMR (200 MHz, DMSO-d 6 ) $\delta$, ppm: 4.68 (s, 2H, $\left.\mathrm{CH}_{2}\right), 6.18$ (bs, 2H, NH $\left.\mathrm{N}_{2}\right), 6.55$ (d, $\left.J=8.7 \mathrm{~Hz}, 2 \mathrm{H}, \mathrm{Ar}-\mathrm{H}\right)$, $7.25(\mathrm{~d}, J=8.7 \mathrm{~Hz}, 2 \mathrm{H}, \mathrm{Ar}-\mathrm{H}), 7.40$ (d, $J=8.7 \mathrm{~Hz}, 2 \mathrm{H}$, Ar-H), 8.17 (d, $J=8.7 \mathrm{~Hz}, 2 \mathrm{H}, \mathrm{Ar}-\mathrm{H})$.

${ }^{13} \mathrm{C}$ NMR (50 MHz, DMSO-d 6$) \delta$, ppm: $38.7\left(\mathrm{CH}_{2}\right)$, $113.0(2 \mathrm{CH}), 123.0(\mathrm{C}), 123.6(2 \mathrm{CH}), 130.5(2 \mathrm{CH})$, $132.6(2 \mathrm{CH}), 137.9(\mathrm{C}), 147.7(\mathrm{C}), 154.3(\mathrm{C})$.

Calculated for $\left(\mathrm{C}_{13} \mathrm{H}_{12} \mathrm{~N}_{2} \mathrm{O}_{4} \mathrm{~S}_{2}\right), \%$ : C 48.14; $\mathrm{H} 3.73$; N 8.64; S 19.77.

Found, \%: C 48.56; H 3.91; N 8.75; S 19.35.

4-Aminobenzenethiosulfonic acid S-(4,5-dimethoxy2-nitrobenzyl) ester (3 b)

Brown precipitate, Mp. $107-109{ }^{\circ} \mathrm{C}$, yield $20 \%$.

${ }^{1} \mathrm{H}$ NMR (200 MHz, DMSO-d $\left.{ }_{6}\right) \delta$, ppm: 3.83 (s, 6H, $\left.\mathrm{OCH}_{3}\right), 4.46\left(\mathrm{~s}, 2 \mathrm{H}, \mathrm{CH}_{2}\right), 5.74\left(\mathrm{~s}, 2 \mathrm{H}, \mathrm{NH}_{2}\right), 6.59(\mathrm{~d}, J=$ $8.8 \mathrm{~Hz}, 2 \mathrm{H}, \mathrm{Ar}-\mathrm{H}), 6.82$ (s, 1H, Ar-H), 7.50 (s, 1H, ArH), 7.52 (s, 1H, Ar-H), 7.57 (s, 1H, Ar-H).

${ }^{13} \mathrm{C}$ NMR (50 MHz, DMSO-d $\left.\mathrm{d}_{6}\right) \delta$, ppm: $37.8\left(\mathrm{CH}_{2}\right)$, $56.5\left(\mathrm{OCH}_{3}\right), 56.7\left(\mathrm{OCH}_{3}\right), 108.7(\mathrm{CH}), 113.0(2 \mathrm{CH})$, $114.4(\mathrm{CH}), 126.6(\mathrm{C}), 129.1(\mathrm{C}), 129.8(2 \mathrm{CH}), 140.2$ (C), 148.5 (C), $153.3(\mathrm{C}), 154.6(\mathrm{C})$.

Calculated for $\left(\mathrm{C}_{15} \mathrm{H}_{16} \mathrm{~N}_{2} \mathrm{O}_{6} \mathrm{~S}_{2}\right), \%$ : C 46.87; H 4.19; N 7.29; S 16.68.

Found, \%: C 46.76; H 4.24; N 7.38; S 16.46.

4-Aminobenzenethiosulfonic acid S-(3-chloroquinoxalin-2-yl-methyl) ester (3 c)

Yellow precipitate, Mp. $135-138{ }^{\circ} \mathrm{C}$, yield $29 \%$.

${ }^{1} \mathrm{H}$ NMR $\left(200 \mathrm{MHz}, \mathrm{CDCl}_{3}\right) \delta$, ppm: 4,42 (s, 2H, $\left.\mathrm{CH}_{2}\right) ; 5,47$ (s, 2H, $\left.\mathrm{NH}_{2}\right) ; 6,83-6,89$ (m, 2H, Ar-H); 7,657,74 (m, 2H, Ar-H); 7,75-7,82 (m, 2H, Ar-H); 7,96-8,12 (m, 2H, Ar-H).

${ }^{13} \mathrm{C}$ NMR $\left(50 \mathrm{MHz}, \mathrm{CDCl}_{3}\right) \delta$, ppm: $41.3\left(\mathrm{CH}_{2}\right)$, $114.7(2 \mathrm{CH}), 129.1(\mathrm{CH}), 129.4(\mathrm{CH}), 129.8(2 \mathrm{CH})$, $130.3(\mathrm{CH}), 130.5(\mathrm{CH}), 135.3(\mathrm{C}), 141.6(\mathrm{C}), 141.8(\mathrm{C})$, 148.1 (C), 150.1 (C), 151.9 (C).

Calculated for $\left(\mathrm{C}_{15} \mathrm{H}_{12} \mathrm{ClN}_{3} \mathrm{O}_{2} \mathrm{~S}_{2}\right), \quad \%$ : C 49.24; H 3.31; Cl 9.69; N 11.49; S 17.53.

Found, \%: C 49.71; H 3.62; N 11.58; S 17.97.

\section{4-Aminobenzenethiosulfonic acid S-(3-methoxy-} quinoxalin-2-ylmethyl) ester (3 d)

Grey precipitate, Mp. $134-136^{\circ} \mathrm{C}$, yield $26 \%$.

${ }^{1} \mathrm{H}$ NMR $\left(200 \mathrm{MHz}, \mathrm{DMSO}-\mathrm{d}_{6}\right) \delta$, ppm: 3,98 (s, 3H, $\left.\mathrm{OCH}_{3}\right) ; 4,36\left(\mathrm{~s}, 2 \mathrm{H}, \mathrm{CH}_{2}\right) ; 6,09\left(\mathrm{~s}, 2 \mathrm{H}, \mathrm{NH}_{2}\right) ; 6,62-6,68$ (m, 2H, Ar-H); 7,69-7,77 (m, 1H, Ar-H); 7,72-7,79 (m, $2 \mathrm{H}, \mathrm{Ar}-\mathrm{H}) ; 7,75-7,88$ (m, 2H, Ar-H), 8,03-8,08 (m, 1H, Ar-H).

${ }^{13} \mathrm{C}$ NMR (50 MHz, DMSO-d 6 ) $\delta$, ppm: $36.4\left(\mathrm{CH}_{2}\right)$, $55.6\left(\mathrm{CH}_{3}\right), 113.9(2 \mathrm{CH}), 126.8(\mathrm{CH}), 129.2(2 \mathrm{CH})$, $129.5(\mathrm{CH}), 130.1(\mathrm{CH}), 131.7(\mathrm{CH}), 135.8(\mathrm{C}), 137.2$ (C), 139.1 (C), 143.7 (C), 152.4 (C), 160.4 (C).

Calculated for $\left(\mathrm{C}_{16} \mathrm{H}_{15} \mathrm{~N}_{3} \mathrm{O}_{3} \mathrm{~S}_{2}\right), \%$ : C 53.17; $\mathrm{H} 4.18$; N 11.63; S 17.74.

Found, \%: C 53.23; H 4.09; N 11.47; S 17.58.
4-Aminobenzenethiosulfonic acid S,S'-[quinoxaline2,3-diylbis(methylene)] ester (3 e)

Grey precipitate, Mp. $152-155^{\circ} \mathrm{C}$, yield $25 \%$.

${ }^{1} \mathrm{H}$ NMR (200 MHz, DMSO-d 6 ) $\delta$, ppm: 4.68 (s, 4H, $\left.2 \mathrm{CH}_{2}\right), 6.33\left(\mathrm{~s}, 4 \mathrm{H}, 2 \mathrm{NH}_{2}\right), 6.53(\mathrm{~s}, 4 \mathrm{H}, \mathrm{Ar}-\mathrm{H}), 7.48$ (s, 4H, Ar-H), 7.87 (m, 4H, Ar-H).

${ }^{13} \mathrm{C}$ NMR (50 MHz, DMSO-d $\left.{ }_{6}\right) \delta$, ppm: $40.5\left(2 \mathrm{CH}_{2}\right)$, $112.9(4 \mathrm{CH}), 126.4(2 \mathrm{CH}), 128.7(2 \mathrm{CH}), 129.7(4 \mathrm{CH})$, 130.9 (2C), 140.4 (2C), 149.3 (2C), $154.6(2 \mathrm{C})$.

Calculated for $\left(\mathrm{C}_{22} \mathrm{H}_{20} \mathrm{~N}_{4} \mathrm{O}_{4} \mathrm{~S}_{4}\right), \%$ : C 49.61; H 3.78; N 10.52; S 24.08.

Found, \%: C 49.75; H 3.62; N 10.43; S 24.21.

4-Aminobenzenethiosulfonic acid S-(1-methyl-4,9dioxo-4,9-dihydro-1H-benzo[f]indol-3-ylmethyl) ester (3 f) $32 \%$.

Grey-yellow precipitate, Mp. 204-207 ${ }^{\circ} \mathrm{C}$, yield

${ }^{1} \mathrm{H}$ NMR (200 MHz, DMSO-d $\left.{ }_{6}\right) \delta$, ppm: 3.85 (s, 3H, $\left.\mathrm{N}-\mathrm{CH}_{3}\right), 4.30\left(\mathrm{~s}, 2 \mathrm{H}, \mathrm{CH}_{2}\right), 6.55(\mathrm{~d}, J=8.8 \mathrm{~Hz}, 2 \mathrm{H}, \mathrm{Ar}-$ H), 7.00 (s, 1H, Ar-H), 7.44 (d, $J=8.8 \mathrm{~Hz}, 2 \mathrm{H}, \mathrm{Ar}-\mathrm{H})$, 7.71-7.78 (m, 2H, Ar-H), 7.91-7.97 (m, 2H, Ar-H).

${ }^{13} \mathrm{C}$ NMR $\left(50 \mathrm{MHz}\right.$, DMSO-d $\left.{ }_{6}\right) \delta$, ppm: $30.5\left(\mathrm{CH}_{2}\right)$, $36.4\left(\mathrm{CH}_{3}\right), 112.5(2 \mathrm{CH}), 118.3(\mathrm{C}), 123.8(\mathrm{C}), 126.0$ $(\mathrm{CH}), 126.1(\mathrm{CH}), 129.4(2 \mathrm{CH}), 130.2(\mathrm{C}), 132.7(\mathrm{C})$, $133.2(\mathrm{CH}), 133.3(\mathrm{CH}), 133.5(\mathrm{CH}), 133.6(\mathrm{C}), 154.2$ (C), $175.0(\mathrm{CO}), 180.7(\mathrm{CO})$.

Calculated for $\left(\mathrm{C}_{20} \mathrm{H}_{16} \mathrm{~N}_{2} \mathrm{O}_{4} \mathrm{~S}_{2}\right), \%$ : C 58.24; H 3.91; N 6.79; S 15.55.

Found, \%: C 58.32; H 3.97; N 6.72; S 15.69.

4-Aminobenzenethiosulfonic acid S-(1-methyl-4,9dioxo-4,9-dihydro-1H-benzo[f]indol-2-ylmethyl) ester (3 g)

Yellow precipitate, Mp. $183-185{ }^{\circ} \mathrm{C}$, yield $70 \%$.

${ }^{1} \mathrm{H}$ NMR (200 MHz, DMSO-d $\left.{ }_{6}\right) \delta$, ppm: 3.89 (s, 3H, $\left.\mathrm{N}-\mathrm{CH}_{3}\right), 4.46$ (s, 2H, $\left.\mathrm{CH}_{2}\right), 6.34$ (br.s, $\left.2 \mathrm{H}, \mathrm{NH}_{2}\right), 6,52$ (s, $1 \mathrm{H}, \mathrm{Ar}-\mathrm{H}), 6.58$ (d, $J=8.6 \mathrm{~Hz}, 2 \mathrm{H}, \mathrm{Ar}-\mathrm{H}), 7.50$ (d, $J=$ 8.6 Hz, 2H, Ar-H), 7.77-7.81 (m, 2H, Ar-H), 7.99-8.07 (m, 2H, Ar-H).

${ }^{13} \mathrm{C}$ NMR $\left(50 \mathrm{MHz}, \mathrm{DMSO}-\mathrm{d}_{6}\right) \delta$, ppm: $28.2\left(\mathrm{CH}_{2}\right)$, $33.9\left(\mathrm{~N}-\mathrm{CH}_{3}\right), 104.0(\mathrm{CH}), 114.3(2 \mathrm{CH}), 126.3(\mathrm{C}), 127.6$ (CH), $127.9(\mathrm{CH}), 129.8(2 \mathrm{CH}), 131.2(\mathrm{C}), 131.9(\mathrm{C})$, $133.5(\mathrm{C}), 134.7(\mathrm{C}), 134.9(\mathrm{CH}), 135.2(\mathrm{CH}), 135.7(\mathrm{C})$, $152.4(\mathrm{C}), 174.6(\mathrm{CO}), 181.2(\mathrm{CO})$.

Calculated for $\left(\mathrm{C}_{20} \mathrm{H}_{16} \mathrm{~N}_{2} \mathrm{O}_{4} \mathrm{~S}_{2}\right), \%$ : C 58.24; H 3.91; N 6.79; S 15.55.

Found, \%: C 58.37; H 4.02; N 6.73; S 15.72.

4-Aminobenzenethiosulfonic acid S-(1,4-dimethoxy9,10-dioxo-9,10-dihydroanthracen-2-ylmethyl) ester (3 h)

Yellow precipitate, Mp. $215-217^{\circ} \mathrm{C}$, yield $30 \%$.

${ }^{1} \mathrm{H}$ NMR (200 MHz, DMSO-d $\left.{ }_{6}\right) \delta$, ppm: $3.83(\mathrm{~s}, 3 \mathrm{H}$, $\left.\mathrm{OCH}_{3}\right), 3.95\left(\mathrm{~s}, 3 \mathrm{H}, \mathrm{OCH}_{3}\right), 4.35\left(\mathrm{~s}, 2 \mathrm{H}, \mathrm{CH}_{2}\right), 6.09(\mathrm{~s}$, $\left.2 \mathrm{H}, \mathrm{NH}_{2}\right), 6.69$ (d, $\left.J=8.6 \mathrm{~Hz}, 2 \mathrm{H}, \mathrm{Ar}-\mathrm{H}\right), 7.28(\mathrm{~s}, 1 \mathrm{H}$, Ar-H), 7.67 (d, 2H, J=8.6 Hz, Ar-H), 7.71-7.74 (m, 2H, Ar-H), 8.12-8.17 (m, 2H, Ar-H).

${ }^{13} \mathrm{C}$ NMR (50 MHz, DMSO-d 6 ) $\delta$, ppm: $34.5\left(\mathrm{CH}_{2}\right)$, $56.8\left(\mathrm{OCH}_{3}\right), 62.7\left(\mathrm{OCH}_{3}\right), 114.0(2 \mathrm{CH}), 120.4(\mathrm{CH})$, $125.4(\mathrm{C}), 126.4(\mathrm{CH}), 126.6(\mathrm{CH}), 127.1(\mathrm{C}), 129.6$ 
(CH), $133.0(\mathrm{C}), 133.4(\mathrm{CH}), 133.6(\mathrm{C}), 133.8(2 \mathrm{CH})$, 134.2 (C), 138.3 (C), 151.1 (C), 152.3 (C), 156.2 (C), 182.7 (CO), 183.0 (CO).

Calculated for $\left(\mathrm{C}_{23} \mathrm{H}_{19} \mathrm{NO}_{6} \mathrm{~S}_{2}\right), \%$ : C 58.84; $\mathrm{H} 4.08$; N 2.98; S 13.66.

Found, \%: C 58.02; H 4.41; N 2.76; S 12.85.

4-Aminobenzenethiosulfonic acid S,S'-[(1,4-dimethoxy-9,10-dioxo-9,10-dihydroanthracene-2,3-diyl)bis(methylene)] ester ( 3 i)

Yellow precipitate, Mp. $121-124{ }^{\circ} \mathrm{C}$, yield $40 \%$.

${ }^{1} \mathrm{H}$ NMR (200 MHz, DMSO-d $\left.{ }_{6}\right) \delta$, ppm: 3.64 (s, 6H, $\left.2 \mathrm{OCH}_{3}\right), 4.32\left(\mathrm{~s}, 4 \mathrm{H}, 2 \mathrm{CH}_{2}\right), 6.12\left(\mathrm{~s}, 4 \mathrm{H}, 2 \mathrm{NH}_{2}\right), 6.62-$ 6.69 (m, 4H, Ar-H), 7.72-7.78 (m, 4H, Ar-H), 7.84-7.88 (m, 2H, Ar-H), 8.04-8.07 (m, 2H, Ar-H).

${ }^{13} \mathrm{C}$ NMR $\left(50 \mathrm{MHz}, \mathrm{DMSO}-\mathrm{d}_{6}\right) \delta$, ppm: $27.3\left(2 \mathrm{CH}_{2}\right)$, $61.8\left(2 \mathrm{CH}_{3}\right), 113.9(4 \mathrm{CH}), 122.8(2 \mathrm{C}), 126.7(2 \mathrm{CH})$, $129.4(2 \mathrm{CH}), 134.9(2 \mathrm{CH}), 135.6(2 \mathrm{C}), 135.9(2 \mathrm{C}), 135.8$ (2C), 152.6 (2C), $154.8(2 \mathrm{C}), 182.3$ (2CO).

Calculated for $\left(\mathrm{C}_{30} \mathrm{H}_{26} \mathrm{~N}_{2} \mathrm{O}_{8} \mathrm{~S}_{4}\right), \%$ : C 53.72; H 3.91; N 4.18; S 19.12.

Found, \%: C 53.57; H 3.84; N 4.24; S 18.89.

General procedure for the synthesis of 4-acetylaminobenzenethiosulfonic acid $\mathrm{S}$-(R-methyl) esters $4 \mathbf{a}-\mathbf{i}$

Into a two-necked flask equipped with nitrogen inlet, a solution of appropriate methyl halogenated derivatives $\mathbf{1} \mathbf{a}-\mathbf{i}$ in DMF and dissolved in portions of DMF sodium salt of 4-acetylaminobenzenethiosulfonic acid ( 2 b) was added. The solution was stirred and kept at room temperature for 5 hours. After this time, a TLC analysis showed that compound 2 had been totally consumed. The reaction mixture was treated with ice water and extracted 3 times with dichloromethane. The organic phase was washed with water and then dried over anhydrous sodium sulphate. After evaporation, the product was purified by silica gel chromatography and recrystallized from ethanol and gave the corresponding 4-acetylaminobenzenethiosulfonic acid S-(R-methyl) esters $4 \mathbf{a}-\mathbf{i}$.

By these procedure compounds 4 a-i were synthesized.

4-Acetylaminobenzenethiosulfonic acid S-(4-nitrobenzyl) ester ( 4 a)

White precipitate, Mp $170-173{ }^{\circ} \mathrm{C}$, yield $60 \%$.

${ }^{1} \mathrm{H}$ NMR (200 MHz, DMSO-d $\left.\mathrm{d}_{6}\right) \delta$, ppm: $2.09(\mathrm{~s}, 3 \mathrm{H}$, $\left.\mathrm{CH}_{3}\right), 4.85\left(\mathrm{~s}, 2 \mathrm{H}, \mathrm{CH}_{2}\right), 7.42(\mathrm{~d}, J=8.7 \mathrm{~Hz}, 2 \mathrm{H}, \mathrm{Ar}-\mathrm{H})$, $7.61(\mathrm{~d}, J=8.8 \mathrm{~Hz}, 2 \mathrm{H}, \mathrm{Ar}-\mathrm{H}), 7.76(\mathrm{~d}, J=8.8 \mathrm{~Hz}, 2 \mathrm{H}$, Ar-H), 8.19 (d, J=8.7 Hz, 2H, Ar-H), 10.41 (s, 1H, NH).

${ }^{13} \mathrm{C}$ NMR $\left(50 \mathrm{MHz}, \mathrm{DMSO}-\mathrm{d}_{6}\right) \delta$, ppm: $24.6\left(\mathrm{CH}_{3}\right)$, $38.3\left(\mathrm{CH}_{2}\right), 119.8(2 \mathrm{CH}), 124.3(2 \mathrm{CH}), 129.4(2 \mathrm{CH})$, $129.5(2 \mathrm{CH}), 139.0(\mathrm{C}), 142.6(\mathrm{CH}), 144.3(\mathrm{C}), 147.3$ (C), 169.5 (CO).

Calculated for $\left(\mathrm{C}_{15} \mathrm{H}_{14} \mathrm{~N}_{2} \mathrm{O}_{5} \mathrm{~S}_{2}\right), \%$ : C 49.17; H 3.85; N 7.65; S 17.50 .

Found, \%: C 49.28; H 3.72; N 7.53; S 17.58.

4-Acetylaminobenzenethiosulfonic acid S-(4,5-dimethoxy-2-nitrobenzyl) ester (4 b)

Yellow precipitate, Mp. $176-178{ }^{\circ} \mathrm{C}$, yield $65 \%$.
${ }^{1} \mathrm{H}$ NMR $\left(200 \mathrm{MHz}, \mathrm{CDCl}_{3}\right) \delta$, ppm: $2.22(\mathrm{~s}, 3 \mathrm{H}$, $\left.\mathrm{CH}_{3}\right), 3.94\left(\mathrm{~s}, 6 \mathrm{H}, 2 \mathrm{OCH}_{3}\right), 4.53\left(\mathrm{~s}, 2 \mathrm{H}, \mathrm{CH}_{2}\right), 6.87$ (s, 1H, NH), 7.62-7.77 (m, 6H, Ar-H).

${ }^{13} \mathrm{C} \mathrm{NMR}\left(50 \mathrm{MHz}, \mathrm{CDCl}_{3}\right) \delta$, ppm: $24.8\left(\mathrm{CH}_{3}\right), 38.5$ $\left(\mathrm{CH}_{2}\right), 56.4\left(\mathrm{OCH}_{3}\right), 56.6\left(\mathrm{OCH}_{3}\right), 108.3(\mathrm{CH}), 113.7$ $(\mathrm{CH}), 119.1(2 \mathrm{CH}), 126.1(\mathrm{C}), 128.3(2 \mathrm{CH}), 139.2(\mathrm{C})$, 140.0 (C), 142.8 (C), 148.7 (C), 153.3 (C), 168.7 (CO).

Calculated for $\left(\mathrm{C}_{17} \mathrm{H}_{18} \mathrm{~N}_{2} \mathrm{O}_{7} \mathrm{~S}_{2}\right), \%$ : C 47.88; $\mathrm{H} 4.25$; N 6.57; S 15.04.

Found, \%: C 47.80; H 4.14; N 6.45; S 15.16.

4-Acetylaminobenzenethiosulfonic acid S-(3-chloroquinoxalin-2-yl-methyl) ester (4 c)

White precipitate, Mp. $175-179^{\circ} \mathrm{C}$, yield $50 \%$.

${ }^{1} \mathrm{H}$ NMR (200 MHz, $\left.\mathrm{CDCl}_{3}\right) \delta$, ppm: $2.20(\mathrm{~s}, 3 \mathrm{H}$, $\left.\mathrm{CH}_{3}\right), 4.70\left(\mathrm{~s}, 2 \mathrm{H}, \mathrm{CH}_{2}\right), 7.53(\mathrm{~d}, J=8.9 \mathrm{~Hz}, 2 \mathrm{H}, \mathrm{Ar}-\mathrm{H})$, 7.74-7.79 (m, 2H, Ar-H), 7.86 (d, $J=\mathrm{Hz}, 2 \mathrm{H}$, Ar-H), 7.94-7.99 (m, 2H, Ar-H).

${ }^{13} \mathrm{C}$ NMR (50 MHz, $\left.\mathrm{CDCl}_{3}\right) \delta$, ppm: $24.5\left(\mathrm{CH}_{3}\right), 41.3$ $\left(\mathrm{CH}_{2}\right), 121.8(2 \mathrm{CH}), 128.6(\mathrm{CH}), 128.7(2 \mathrm{CH}), 128.9$ $(\mathrm{CH}), 130.1(\mathrm{CH}), 130.3(\mathrm{CH}), 138.8(\mathrm{C}), 140.4(\mathrm{C})$, 141.8 (C), 143.7 (C), 147.9 (C), 149.8 (C), 168.7 (CO).

Calculated for $\left(\mathrm{C}_{17} \mathrm{H}_{14} \mathrm{~N}_{3} \mathrm{O}_{3} \mathrm{~S}_{2} \mathrm{Cl}\right), \%$ : C 50.06;

$\mathrm{H} 3.46$; N 10.30; S 15.72; Cl 8.69.

Found, \%: C 50.21; H 3.52; N 10.38; S 15.74 .

4-Acetylaminobenzenethiosulfonic acid S-(3methoxyquinoxalin-2-ylmethyl) ester (4 d)

Pink precipitate, Mp. $78-80{ }^{\circ} \mathrm{C}$, yield $50 \%$.

${ }^{1} \mathrm{H}$ NMR (200 MHz, DMSO-d 6 ) $\delta$, ppm: 2.06 (s, 3H, $\left.\mathrm{CH}_{3}\right), 4.01\left(\mathrm{~s}, 3 \mathrm{H}, \mathrm{OCH}_{3}\right), 4.56\left(\mathrm{~s}, 2 \mathrm{H}, \mathrm{CH}_{2}\right), 7.40-7.88$ (m, 8H, Ar-H), 10.28 (s, 1H, NH).

${ }^{13} \mathrm{C}$ NMR $\left(50 \mathrm{MHz}, \mathrm{DMSO}-\mathrm{d}_{6}\right) \delta$, ppm: $24.6\left(\mathrm{CH}_{3}\right)$, $36.4\left(\mathrm{CH}_{2}\right), 55.5\left(\mathrm{OCH}_{3}\right), 119.7(2 \mathrm{CH}), 126.9(\mathrm{CH})$, $129.3(2 \mathrm{CH}), 129.5(\mathrm{CH}), 129.9(\mathrm{CH}), 131.6(\mathrm{CH}), 136.9$ (C), 139.3 (C), 139.4 (C), 143.4 (C), 144.3 (C), 160.2 (C), $169.6(\mathrm{CO})$.

Calculated for $\left(\mathrm{C}_{18} \mathrm{H}_{17} \mathrm{~N}_{3} \mathrm{O}_{4} \mathrm{~S}_{2}\right), \%$ : C 53.58; H 4.25; N 10.41; S 15.89.

Found, \%: C 53.65; H 4.31; N 10.47; S 15.95.

4-Acetylaminobenzenethiosulfonic acid S,S'[quinoxaline-2,3-diylbis(methylene)] ester (4 e)

White precipitate, Mp. $163-167{ }^{\circ} \mathrm{C}$, yield $40 \%$.

${ }^{1} \mathrm{H}$ NMR (200 MHz, DMSO-d $\left.{ }_{6}\right) \delta$, ppm: 2.07 (s, 6H, $\left.2 \mathrm{CH}_{3}\right), 4.77\left(\mathrm{~s}, 4 \mathrm{H}, 2 \mathrm{CH}_{2}\right), 7.59-7.77(\mathrm{~m}, 12 \mathrm{H}, \mathrm{Ar}-\mathrm{H})$, 10.29 (s, 2H, 2NH).

${ }^{13} \mathrm{C}$ NMR $\left(50 \mathrm{MHz}, \mathrm{DMSO}-\mathrm{d}_{6}\right) \delta$, ppm: $24.4\left(2 \mathrm{CH}_{3}\right)$, $39.7\left(2 \mathrm{CH}_{2}\right), 118.5(4 \mathrm{CH}), 128.2(2 \mathrm{CH}), 128.3(2 \mathrm{CH})$, 130.5 (4CH), 137.3 (2C), 139.8 (2C), 144.3 (2C), 148.5 (2C), 169.2 (2CO).

Calculated for $\left(\mathrm{C}_{26} \mathrm{H}_{24} \mathrm{~N}_{4} \mathrm{O}_{6} \mathrm{~S}_{4}\right), \%$ : C 50.63; H 3.92; N 9.08; S 20.80.

Found, \%: C 50.75; H 3.86; N 9.02; S 20.89.

4-Acetylaminobenzenethiosulfonic acid S-(1-methyl4,9-dioxo-4,9-dihydro-1H-benzo[f]indol-3-ylmethyl) ester (4 f)

Yellow precipitate, Mp. $224-227{ }^{\circ} \mathrm{C}$, yield $70 \%$.

${ }^{1} \mathrm{H}$ NMR (200 MHz, DMSO-d 6 ) $\delta$, ppm: 2.07 (s, 3H, $\left.\mathrm{CH}_{3}\right), 3.87\left(\mathrm{~s}, 3 \mathrm{H}, \mathrm{N}-\mathrm{CH}_{3}\right), 4.44\left(\mathrm{~s}, 2 \mathrm{H}, \mathrm{CH}_{2}\right), 7.15$ (s, 
1H, Ar-H), 7.68 (d, J=4.4 Hz, 4H, Ar-H), 7.78-7.82 (m, 2H, Ar-H), 7.97-8.04 (m, 2H, Ar-H), 10.33 (s, 1H, NH).

${ }^{13} \mathrm{C}$ NMR $\left(50 \mathrm{MHz}, \mathrm{DMSO}-\mathrm{d}_{6}\right) \delta$, ppm: $24.4\left(\mathrm{CH}_{3}\right)$, $31.0\left(\mathrm{CH}_{2}\right), 36.4\left(\mathrm{~N}-\mathrm{CH}_{3}\right), 117.6(\mathrm{C}), 118.3(2 \mathrm{CH}), 123.8$ (C), $126.0(\mathrm{CH}), 126.1(\mathrm{CH}), 128.4(\mathrm{CH}), 130.3(\mathrm{C})$, $133.0(\mathrm{CH}), 133.2(\mathrm{C}), 133.4(\mathrm{C}), 133.7(\mathrm{CH}), 133.8$ $(\mathrm{CH}), 137.7(\mathrm{C}), 144.2(\mathrm{C}), 169.3(\mathrm{C}(\mathrm{O}) \mathrm{N}), 175.1(\mathrm{CO})$, 180.7 (CO).

Calculated for $\left(\mathrm{C}_{22} \mathrm{H}_{18} \mathrm{~N}_{2} \mathrm{O}_{5} \mathrm{~S}_{2}\right), \%$ : C 58.14; H 3.99; N 6.16; S 14.11.

Found, \%: C 58.43; H 3.82; N 6.37; S 14.03.

4-Acetylaminobenzenethiosulfonic acid S-(1-methyl4,9-dioxo-4,9-dihydro-1H-benzo[f]indol-2-ylmethyl) ester (4 g)

Yellow precipitate, Mp. $208-211^{\circ} \mathrm{C}$, yield $55 \%$.

${ }^{1} \mathrm{H}$ NMR (200 MHz, DMSO-d 6 ) $\delta$, ppm: $1.82(\mathrm{~s}, 3 \mathrm{H}$, $\left.\mathrm{CH}_{3}\right), 3.87\left(\mathrm{~s}, 3 \mathrm{H}, \mathrm{N}-\mathrm{CH}_{3}\right), 4.57\left(\mathrm{~s}, 2 \mathrm{H}, \mathrm{CH}_{2}\right), 6.40(\mathrm{~s}$, 1H, Ar-H), 7.56 (d, $J=8.9 \mathrm{~Hz}, 2 \mathrm{H}, \mathrm{Ar}-\mathrm{H}), 7.67$ (d, $J=$ 8.9 Hz, 2H, Ar-H), 7.76-7.80 (m, 2H, Ar-H), 7.96-8.02 (m, 2H, Ar-H), $10.13(\mathrm{~s}, 1 \mathrm{H}, \mathrm{NH})$.

${ }^{13} \mathrm{C}$ NMR (50 MHz, DMSO-d 6 ) $\delta$, ppm: $24.1\left(\mathrm{CH}_{3}\right)$, $31.0\left(\mathrm{CH}_{2}\right), 33.1\left(\mathrm{~N}-\mathrm{CH}_{3}\right), 109.5(\mathrm{CH}), 118.0(\mathrm{CH}), 118.2$ $(\mathrm{CH}), 126.1(\mathrm{CH}), 126.3(\mathrm{CH}), 126.8(\mathrm{C}), 128.1(2 \mathrm{CH})$, 130.9 (C), 132.9 (C), 133.6 (2CH), 133.7 (C), 135.7 (C), $137.3(\mathrm{C}), 144.3(\mathrm{C}), 168.9(\mathrm{C}(\mathrm{O}) \mathrm{N}), 175.0(\mathrm{CO}), 179.7$ (CO).

Calculated for $\left(\mathrm{C}_{22} \mathrm{H}_{18} \mathrm{~N}_{2} \mathrm{O}_{5} \mathrm{~S}_{2}\right), \%$ : C 58.14; H 3.99; N 6.16; S 14.11.

Found, \%: C 58.37; H 3.78; N 6.26; S 14.23.

4-Acetylaminobenzenethiosulfonic acid S-(1,4-dimethoxy-9,10-dioxo-9,10-dihydroanthracen-2-ylmethyl) ester ( $4 \mathrm{~h})$

Orange precipitate, Mp. $207-212{ }^{\circ} \mathrm{C}$, yield $30 \%$.

${ }^{1} \mathrm{H}$ NMR $\left(200 \mathrm{MHz}, \mathrm{DMSO}-\mathrm{d}_{6}\right) \delta$, ppm: 1,97 (s, 3H, $\left.\mathrm{CH}_{3}\right), 3.70\left(\mathrm{~s}, 3 \mathrm{H}, \mathrm{OCH}_{3}\right), 3.82\left(\mathrm{~s}, 3 \mathrm{H}, \mathrm{OCH}_{3}\right), 4.43(\mathrm{~s}$, $\left.2 \mathrm{H}, \mathrm{CH}_{2}\right), 7.40(\mathrm{~s}, 1 \mathrm{H}, \mathrm{Ar}-\mathrm{H}), 7.68(\mathrm{~d}, J=9.0 \mathrm{~Hz}, 2 \mathrm{H}$, Ar-H), 7.77-7.88 (m, 4H, Ar-H), 7.98-8.06 (m, 2H, Ar$\mathrm{H}), 10,10(\mathrm{~s}, 2 \mathrm{H}, \mathrm{NH})$.

${ }^{13} \mathrm{C}$ NMR $\left(50 \mathrm{MHz}, \mathrm{DMSO}-\mathrm{d}_{6}\right) \delta$, ppm: $24.3\left(\mathrm{CH}_{3}\right)$, $34.6\left(\mathrm{CH}_{2}\right), 56.7\left(\mathrm{OCH}_{3}\right), 62.2\left(\mathrm{OCH}_{3}\right), 118.5(2 \mathrm{CH})$, $121.3(\mathrm{CH}), 126.1(\mathrm{CH}), 126.2(2 \mathrm{CH}), 126.6(\mathrm{C}), 128.5$ (2CH), $133.4(\mathrm{C}), 133.7(\mathrm{CH}), 134.0(\mathrm{CH}), 134.1(\mathrm{C})$, 137.3 (C), 138.0 (C), 144.5 (C), 151.7 (C), 155.5 (C), $169.2(\mathrm{C}), 181.4(\mathrm{CO}), 182.3(\mathrm{CO})$.

Calculated for $\left(\mathrm{C}_{25} \mathrm{H}_{21} \mathrm{NO}_{7} \mathrm{~S}_{2}\right), \%$ : C 58.70; H 4.14; N 2.74; S 12.54 .

Found, \%: C 58.83; H 4.02; N 2.67; S 12.62.

4-Acetylaminobenzenethiosulfonic acid $S, S^{\prime}-[(1,4-$ dimethoxy-9,10-dioxo-9,10-dihydroanthracene-2,3-diyl)bis(methylene)] ester (4 i)

Orange precipitate, Mp. $214-217{ }^{\circ} \mathrm{C}$, yield $22 \%$.

${ }^{1} \mathrm{H}$ NMR (200 MHz, DMSO-d 6 ) $\delta$, ppm: $2.00(\mathrm{~s}, 6 \mathrm{H}$, $\left.2 \mathrm{CH}_{3}\right), 3.63\left(\mathrm{~s}, 6 \mathrm{H}, 2 \mathrm{OCH}_{3}\right), 4.41\left(\mathrm{~s}, 4 \mathrm{H}, 2 \mathrm{CH}_{2}\right), 7.72-$ 7.85 (m, 10H, Ar-H), 8.03 (s, 2H, Ar-H), 10.37 (s, 2H, $2 \mathrm{NH})$.
${ }^{13} \mathrm{C}$ NMR (50 MHz, DMSO-d $\left.\mathrm{d}_{6}\right) \delta$, ppm: $24.6\left(2 \mathrm{CH}_{3}\right)$, $31.7\left(2 \mathrm{CH}_{2}\right), 63.0\left(2 \mathrm{OCH}_{3}\right), 119.0(4 \mathrm{CH}), 126.6(2 \mathrm{CH})$, 128.7 (4CH), $133.7(2 \mathrm{C}), 134.5(2 \mathrm{CH}), 136.3(2 \mathrm{C}), 137.3$ (2C), 144.9 (2C), $155.2(2 \mathrm{C}), 169.6$ (2CO), 181.7 (2CO).

Calculated for $\left(\mathrm{C}_{34} \mathrm{H}_{30} \mathrm{~N}_{2} \mathrm{O}_{10} \mathrm{~S}_{4}\right), \%$ : C 54.10; H 4.01; N 3.71; S 16.99.

Found, \%: C 54.19; H 4.23; N 3.64; S 16.87.

\section{Results and discussion}

In this paper, an approach to the synthesis of new derivatives of quinones and quinoxalines with thiosulfonates is discussed. The implementation of this approach could be carried out by the interaction of the corresponding salts of thiosulfoacids that have properties of S,N-binucleophiles with quinoxaline and quinone derivatives. It is known that the selected compounds are bifunctional molecules that can react with halogen derivatives of quinones and quinoxalines in two alternative ways: by a thiosulfonate fragment and also by an amino group. The direction of the reaction can be determined according to the conditions of the synthesis, and the main factor is the selection of the solvent.

Choosing the conditions of the reaction, we took into account our previous works in the synthesis of compounds with the thiosulfonate "core"[13-16].

Due to the bifunctional properties of the selected binucleophiles, we managed to find the conditions of the reaction that lead to products of S-nucleophilic substitution, so experimentally it was found that when carrying out the reaction in polar solvents $(\mathrm{EtOH}$, $\left(\mathrm{CH}_{3}\right)_{2} \mathrm{CO}$, THF $)$, the products of the reaction - 4-aminobenzenethiosulfonic acid S-(4-nitrobenzyl) ester (3 a) were isolated with a good yield of $35-63 \%$, respectively.

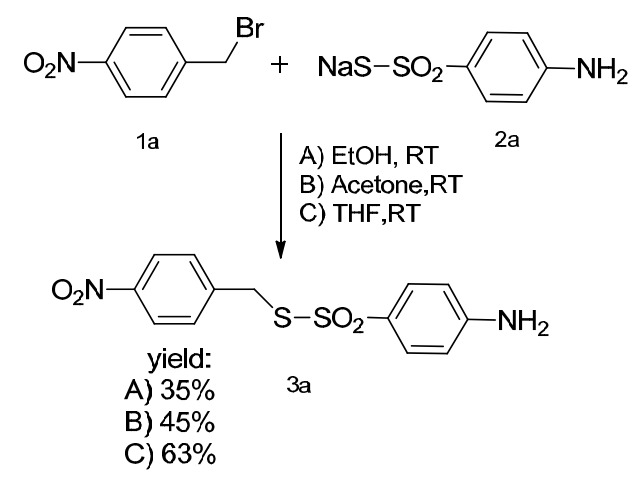

Scheme 1. Optimization of the reaction conditions

Analyzing the optimization data of reaction conditions, the best yield of compound ( 3 a) was obtained in the reaction between sodium salt of 4-aminobenzenethiosulfonic acid ( 2 a) and 1-bromomethyl-4nitrobenzene (1 a) in the THF medium at room temperature for 5 hours. Using the developed method, a number of thiosulfonate derivatives based on quinones and quinoxalines were obtained (Scheme 2). 


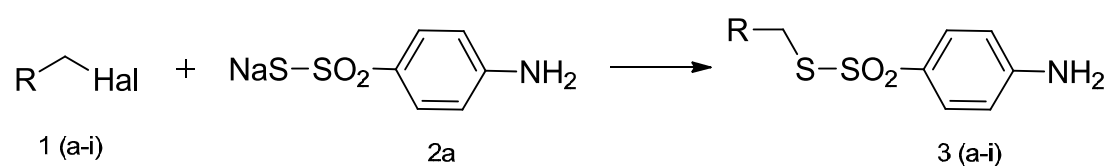

Scheme 2. Synthesis of a number of 4-aminobenzenethiosulfonic acid S-(R-methyl) esters $\mathbf{3}$ a-i

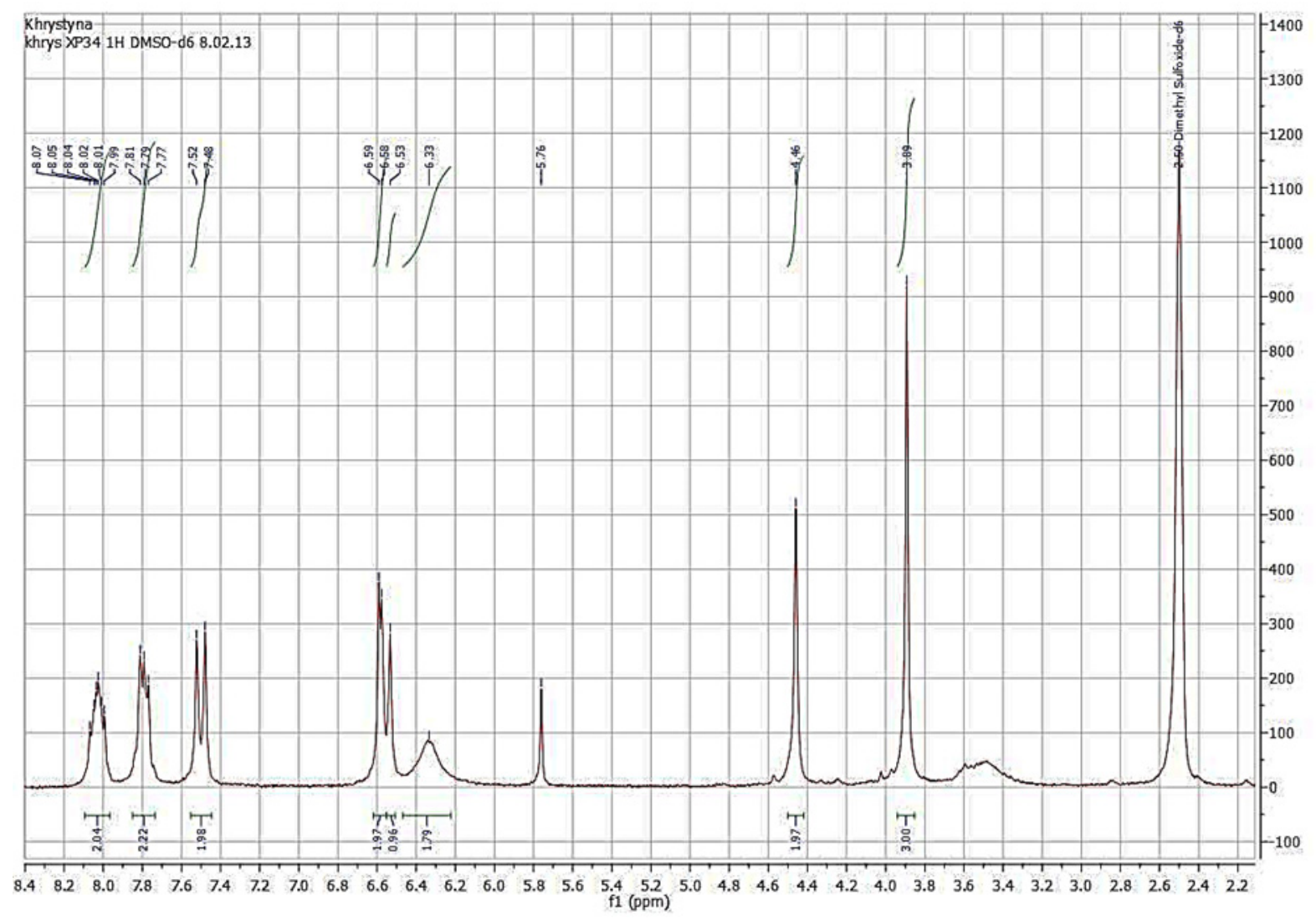

Fig. 1. ${ }^{1} \mathrm{H}$ NMR spectrum of 4-aminobenzenethiosulfonic acid S-(1-methyl-4,9-dioxo-4,9-dihydro-1H-benzo[f]indol-2-ylmethyl) ester (3g) in DMSO-d $\mathrm{d}_{6}$

According to the TLC we detected the formation of the product after 30 minutes. So, on the NMR spectrum of compound (3 $\mathbf{g})$ 4-aminobenzenethiosulfonic acid S-(1-methyl-4,9-dioxo-4,9-dihydro-1H-benzo[f]indol-2ylmethyl) ester, we could observe the signals of a paminobenzenethiosulfonic acid fragment in the aromatic part of the spectrum at $6.52-8.07 \mathrm{ppm}$ and the signals of two protons of the $-\mathrm{NH}_{2}$ group at $6.34 \mathrm{ppm}$ (Fig. 1).
The next step was the optimization of the obtained structures by an electrophilic attack of the free amino group. The possibility of acylation of the products of 4aminobenzenethiosulfonic acid S-(R-methyl) ester 3 a-i using acetyl chloride is shown in the following scheme (path A).

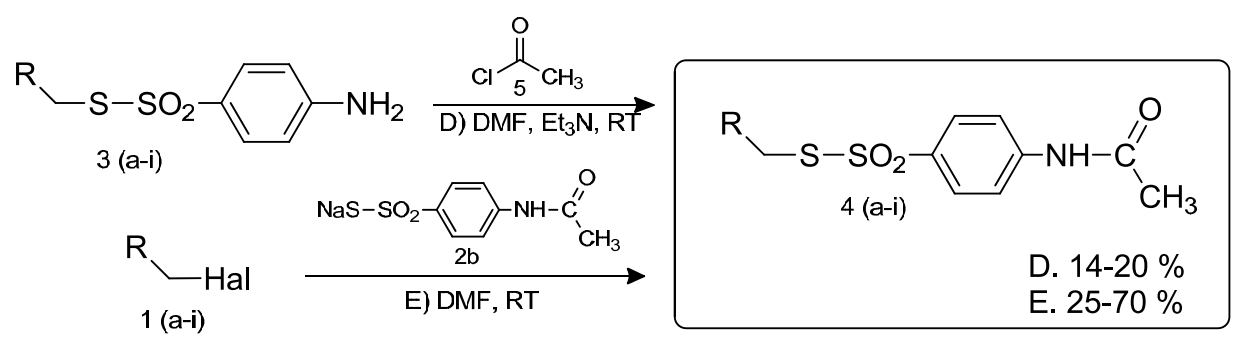

Scheme 3. Synthesis of 4-acetylaminobenzenethiosulfonic acid S-(R-methyl) esters 4 a-i

Derivatives of 4-acetylaminobenzenethiosulfonic acid S-(R-methyl) ester obtained at this stage were isolated with very low yields. So, for the synthesis of acyl derivatives, we took the sodium salt of 4-acetylamino- 
benzenethiosulfonic acid ( 2 b). The use of the already acylated salts of thiosulfoacids that enabled reacting in the stiff conditions (DMF medium) excluded the possibility of the reaction of $\mathrm{N}$-alkylation and allowed obtaining products of 4-acetylaminobenzenethiosulfonic acid S-(R-methyl) ester 4 a-i with the yields of 25-70\% (path B).

These high yields can be explained by using DMF as a solvent, which due to its high polarity causes a rapid dissociation of the $\mathrm{S}-\mathrm{Na}$ bond with the formation of a thiosulfonate-anion and $-\mathrm{CH}_{2}-\mathrm{Cl}$ bond and forming the corresponding carbocation.

The yield of the products of 4-aminobenzenethiosulfonic acid S-(R-methyl) esters 3 a-i and 4-acetylaminobenzenethiosulfonic acid S-(R-methyl) esters 4 a-i is shown in Table 1.

Table 1. The yield of the products of 4-aminobenzenethiosulfonic acid S-(R-methyl) esters 3 a-i and 4-acetylaminobenzenethiosulfonic acid S-(R-methyl) esters $\mathbf{4} \mathbf{a}-\mathbf{i}$

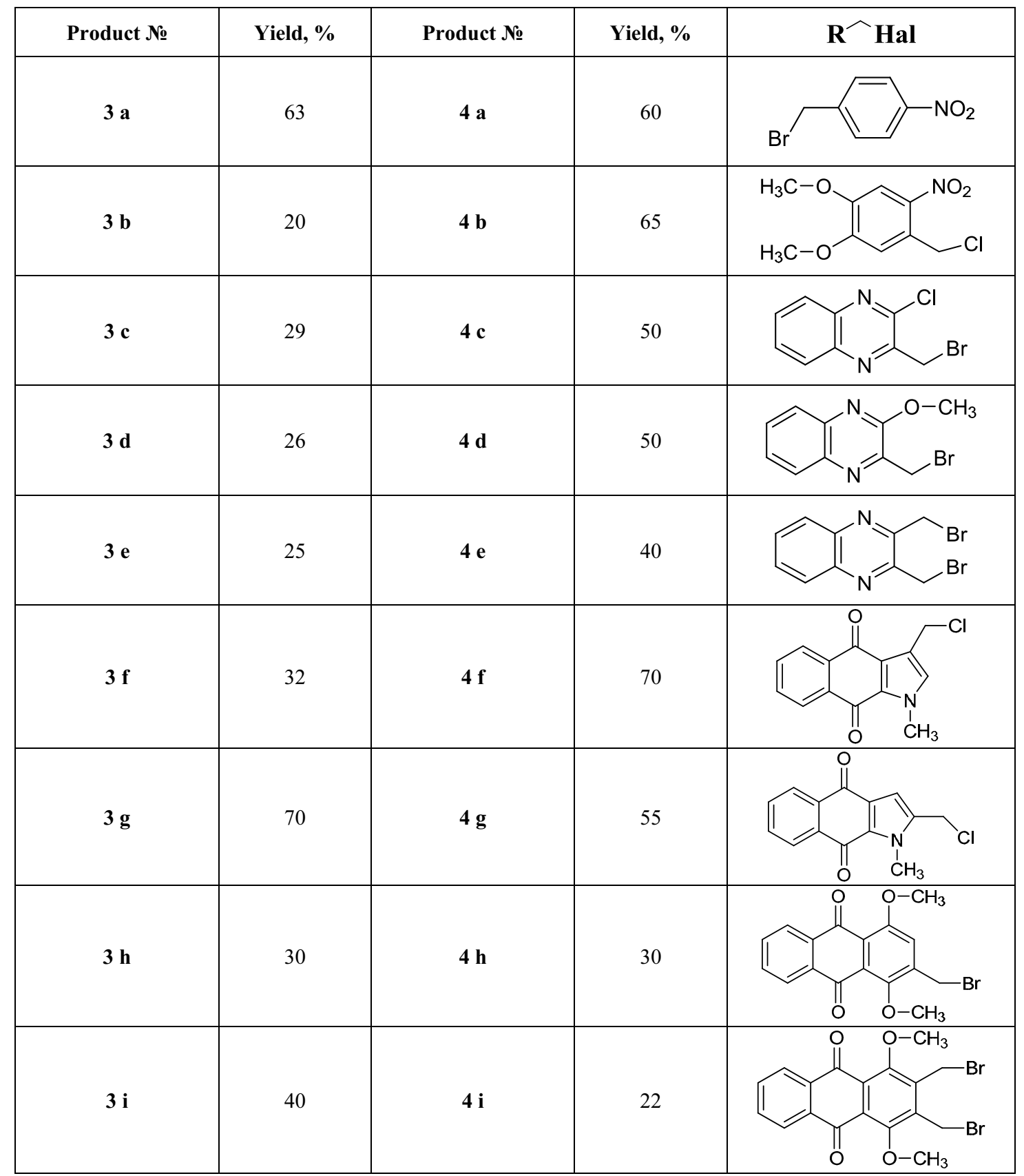

\section{Conclusions}

In this study, we have prepared a number of quinone and quinoxaline derivatives of thiosulfonates using the nucleophilic substitution reaction. We optimised the reaction conditions for the synthesis compounds by a thiosulfonate fragment. The products were obtained in good yields (20-70\%). These studies constitute the first example of the interaction of thiosulfonates with quinones and quinoxalines. 


\section{References}

1. Hayashi S., Furukawa M., Jamamoto J., Hamamura K. The antitumour properties of thiosulfonates // Chem. Pharm. Bull. 1967. Vol. 15. P. $1310-1315$.

http://dx.doi.org/10.1248/cpb.15.1310

2. Lubenets V., Vasylyuk S., Baranovych D., KomarovskaPorokhnyavec O., Rayevska K., Zaichenko O., Novikov $\mathbf{V}$. The plant protection remedies of thiosulfonate type // Chemicals in Agriculture and Environment. 2007. Vol. 8. P. 163-167.

3. Clemendson C. J., Hultman H. J., Sörbo B. The antidote effect of some sulfur compounds and rhodanese in experimental cyanide poisoning // Acta Physiol. Scand. 1954. Vol. 32. P. 245-251. http://dx.doi.org/10.1111/j.1748-1716.1954.tb01171.x

4. Sebille B., Beuzard Y., Demarne H. Pat. FR 2573077, 1984.

5. Nawrot U., Zaczynska E., Czarny A., Lubenets V., Karpenko E. Antifungal activity of synthetic derivatives of allicin - continued research // Mikologia Lekarska. 2012. Vol. 19. N 4. P. 143-146 (in Polish).

6. Petrikovics I., Pei L., McGuinn W. D., Cannon E. P., Way J. L. Encapsulation of rhodanese and organic thiosulfonates by mouse erythrocytes // Fundam. Appl. Toxicol. 1994. Vol. 23. P. 70-75.

http://dx.doi.org/10.1006/faat.1994.1080

7. Pruski A. M., Fiala-Medioni A. Stimulatory effect of sulphide on thiotaurine synthesis in three hydrothermalvent species from the East Pacific Rise // J. Exp. Biol. 2003. Vol. 206. P. 2923-2930. http://dx.doi.org/10.1242/jeb.00513

8. Rosenberg N. K., Lee R. W., Yancey P. H. High contents of hypotaurine and thiotaurine in hydrothermalvent gastropods without thiotrophic endosymbionts // J. Exp. Zool. 2006. Vol. 305A. P. 655-622. http://dx.doi.org/10.1002/jez.a.316

9. Arcamone F. Properties of antitumor anthracyclines and new developments in their application: cain memorial award lecture// Cancer Res. 1985. Vol. 45. P. 5995-5999.

10. Monneret C. Recent developments in the field of antitumour anthracyclines // Eur. J. Med. Chem. 2001. Vol. 36. P. 483-493. http://dx.doi.org/10.1016/S0223-5234(01)01244-2

11. Hazeldine S. T., Polin L., Kushner J., White K., Corbett T. H., Horwitz J. P. Synthesis and biological evaluation of conformationally constrained analogs of the antitumor agents XK469 and SH80 // Bioorganic \& Medicinal Chemistry. 2006. Vol. 14. N 7. P. 2462-2467. http://dx.doi.org/10.1016/j.bmc.2005.11.036
12. Tandon V. K., Yadav D. B., Maurya H. K.,Chaturvedi A. K., Shukla P. K. Design, synthesis, and biological evaluation of 1,2,3-trisubstituted-1,4-dihydrobenzo[g]quinoxaline-5,10-diones and related compounds as antifungal and antibacterial agents // Bioorganic \& Medicinal Chemistry. 2006. Vol. 14. N 17. P. 6120-6126. http://dx.doi.org/10.1016/j.bmc.2006.04.029

13. Lubenets V., Karpenko O., Ponomarenko M., Zahoriy G., Krychkovska A., Novikov V. Development of new antimicrobial compositions of thiosulfonate structure // Chemistry and Chemical Technology. 2013. Vol. 7. N 2. P. 119-124.

14. Lubenets V. I., Vasylyuк S. V., Goy O. V., Novikov V. P. Reaction of 6,7-dichloroquinoline-5,8-quinone with thiosulfonic acid salts // Chemistry of Heterocyclic Compounds. 2006. Vol. 42. N 7. P. 961-962.

15. Lubenets V. I., Vasylyuк S. V., Goy O. V., But S. O., Chernega A. N., Novikov V. P. Synthesis and properties of S-esters of 2,3-dioxo-1,2,3,4-tetrahydroquinoxaline-6thiosulfoacid // Journal of Organic and Pharmaceutical Chemistry. 2006. Vol. 4. P. 56-63 (in Ukrainian).

16. Lubenets V. I., Vasylyuk S. V., Novikov V. P. Synthesis of S-(3-chloroquinoxaline-2-yl)esters of aliphatic and aromatic thiosulfonic acids // Chemistry of Heterocyclic Compounds. 2005. Vol. 41. N 12. P. 1547-1548.

http://dx.doi.org/10.1007/s10593-006-0039-9.

K. Bolibrukh, N. Monka, S. Polovkovych, V. Lubenets, V. Novikov, O. Khoumeri, T. Terme, P. Vanelle, O. Solovyov

\section{CHINONO IR CHINOKSALINO FRAGMENTUS TURINČIŲ TIOSULFONATŲ SINTEZE}

\section{Santra u a}

Susintetinti nauji tiosulfonatai, turintys naftochinono ir chinoksalino fragmentus. Sintezei panaudoti S,N-binukleofilai: 4-amino- ir 4-acetilaminobenzentiosulfoninių rūgščių natrio druskos. Optimizuotos sintezès sąlygos, leidžiančios gerokai padidinti sintetinamų junginių išeigą. 